\title{
On Research Progress and Development Trend for Motion Control Problems of Industrial Robots
}

\author{
Xianxi Luo ${ }^{a}$, Shubo Liu', Menghua Xuc ${ }^{c}$, Junjian $\mathrm{He}^{\mathrm{d}}$ \\ Jiangxi Province Engineering Research Center of New Energy Technology and Equipment \\ (East China University of Technology), Nanchang, Jiangxi, China \\ aXianxi_luo@yahoo.com, b191233558@qq.com, c516894709@qq.com, d409634648@qq.com
}

\begin{abstract}
Keywords: Industrial Robot, Motion Control, Research Progress, Development Trend
Abstract: Robotics is one of the most important technologies that affect human being in the twenty-first Century. Motion control is still the classical and difficult research problem in domain of industrial robot technology. In this paper, the important technical problems and research progress of the motion control of industrial robots are discussed. These problems include the identification of the dynamic parameters, trajectory planning, trajectory tracking and dynamic motion control of industrial robots. This paper also analyzes the direction and development trend of the future researches on these problems to adapt to the requirements of the expanding applications and escalating performances.
\end{abstract}

\section{Introduction}

After entering the twenty-first Century, under environment of rapidly developing technology and the increasing demand of people, robot technology has attracted unprecedented attention. It is regarded as the core of the new technology revolution [1]. Especially in recent years, to cope with the changes in the economic situation and the shortage of labors in the market, many enterprises actively began to upgrade their devices. The International Federation of Robotics (IFR) predicted that in 2018, China's industrial robot sales will reach 180 thousand, accounting for one third in the world. The total amount value of China's industrial robot market will reach 600 billion dollars in the next decade[2]. In April 2016, the Ministry of Industry and Information, Ministry of development and reform and the Ministry of Finance jointly issued the robot industry development plan (2016-2020), and put forward that by 2020, the annual output of autonomous brand industrial robots will reach 100 thousand units. The robot technology and industry have entered a new period of development in China.

From the aspect of long period of development, industrial robots will gradually replace the manual operation, this trend demands the increasing performances of the intelligence, stability, high efficiency, high precision and high speed [3]. The final performance depends on the joint performance of motion control. So, the joint motion control is the fundamental and classical problem to research.

Articulated industrial robot is a typical industrial robot with several joints. It has the properties of time-varying in parameters, strong coupling between different joints and nonlinear dynamic characteristics. It is very difficult to achieve precise and fast control based on mathematical models [4]. Firstly, the establishment of the precise model and identify the model parameters is very difficult because of the measurement errors, the clearance of mechanical transmission and the influence of wear. Secondly, the nonlinear characteristics of the system, the coupling interference between the mechanisms, the computation speed of traditional control strategy and the dynamic characteristics of the mechanism also constrains the extent to improve the control performances[5]. Thirdly, industrial robot cannot predict external disturbances such as load changes, manipulator deformation and motor working conditions, which seriously influence the performance of robots. Independent joint PID servo control is the commonly applied for the general purpose industrial robots. It is difficult to 
satisfy the requirements of the specified trajectory with high speed and high precision in tracking operations.

In recent years, many research institutions and researchers proposed a lot of control strategies such as calculation of torque feedforward control, sliding mode variable structure control, linear quadratic regulator(LQR), robust control, artificial neural network [6] to solve the problem of motion control. However, most of these algorithms have limitations in algorithm complexity, control performances and adaptive conditions. They are usually difficult to be applied as robust algorithms[]].

The researches on motion control of industrial robot involves many aspects. In this paper, three problems, namely the dynamic parameter identification, trajectory planning and trajectory tracking control of industrial robots, of the research progress are to be explored in section 2-4. The development trend and potential solutions of these three problems are presented in section 5. Finally, the conclusion is presented in the end.

\section{Parameter identification}

There are several matrices comprised of the parameters to be identified in the inverse dynamic equations. They are inertia matrix, Coriolis and Centripetal coupling matrix, the friction force matrix and gravity loading matrix. Each element in these matrices are varying with the current pose of the industrial robot. In trajectory tracking control, the computation torque control depends on the precise dynamic model. These parameter identification of the model is the premise of trajectory tracking control. Billings proposed cross correlation decoupling parameter identification method, Gautier proposed inertial parameter minimum set and least squares method, Olsen proposed maximum likelihood method and so on. For the new flexible arm robot and parallel robot, the researchers also put forward the optimization and weighted least square method under the constraint conditions[ㅁ]

In recent years, intelligent algorithms and optimization theories developed rapidly and played a significant role in the research of robot parameter identification. Khakassia put forward the method of dynamic characteristics based on the nonlinear function characteristic of neural network model, and verified it on Stanford six degree of freedom robot. After that, Anh used a new MIMO NARX neural network model with a new inverse dynamic transformation, and trained neural network model by the input and output data to get a better identification precision [9]. Bingul et al. proved that with least square (LS) and particle swarm optimization (PSO) the estimated can predict the dynamic characteristic better[10]. Koker et al. proposed a combined algorithm of neural network and genetic algorithm for parameter identification and inverse kinematics problem Stanford robot [11] . Danping Yan proposed a heuristic particle search algorithm to identify the parameters of industrial robot[12], the author thought the proposed algorithm can better identify the model parameters.

The intelligent algorithm is insensitive to the characteristics of the kinematics model, and can search flexibly in the multidimensional nonlinear space, the advantages is obvious in the application of parameter identification. Although researchers have made many explorations on robot parameter identification, it's difficult to achieve practically because the industrial robot has many parameters, unpredictable load and flexible operation requirements. So far, there is no generally applicable and practical methods.

\section{trajectory planning of industrial robot}

The requirements of peed, stability and accuracy are different for different applications. Because trajectory planning is the key step to ensure the operation standard, it has also become an important research topic in the field of robot. 
The early trajectory planning mainly pursued shorter running time and smoothness, moving speed, acceleration and jerk. Bazaz and Tundou concluded that the simplest curve to connect every point in the joint space is the cubic polynomial curve, and later innovated to quintic polynomial curve to get better accuracy and stability [13]. Since then, a large number of studies focused the problem of the transmission stability and the optimal executive time[14]. Cho et al. proposed to minimize torque and jerk to produce a more smooth motion path [15]. The latest research was aimed at the least energy consumption, and applied the iterative dynamic programming [16] or weighted objective function to optimize the [17] working parameters. To make the algorithm closer to reality, some studies also considered the barriers and joint moving limits $[\underline{18}, \underline{19}]$.

At present, there are many ways to accomplish the trajectory planning for specific tasks within a given time. These algorithms are gradually improved, and the working conditions are set to the real environment. However, the online trajectory planning is still lack of practical application. Besides, there are limited methods and techniques to minimize the trajectory execution time, energy consumption and other types of multi-objective optimization.

\section{Trajectory tracking control}

The trajectory tracking control for industrial robots is the dynamic control of the joints. Dynamic trajectory control based on Newton_Euler or Lagrangian energy equation was the integration of research results before 1985, and became a classical method of robot joint dynamic control. However, the calculation of this method was very large, and it was difficult to be applied in the computer at that time. Armstrong and Luh et al. put forward the method of repeatively training and iterative computation in real time, so that the amount of computation was greatly reduced. These studies became the basis of the trajectory tracking control of the robot [20]. There were two major problems that restrict the application of Newton_Euler or computational torque control methods, namely, the parameter errors of the model and the uncertain factors that affect the operation of the robot[]. The ideal control performance cannot be obtained through the calculated torque feedforward control alone.

Researchers tried many new control methods for different applications. The PID control of independent joints cannot overcome so many disturbances to achieve high control accuracy. Ajwad and Fei Y designed intelligent PID controller to improve the control effect by optimizing the parameters, improving the measurement and cascading multi-controller [21, 22]. The hierarchical intelligent control structure, Particle Swarm Optimization (PSO), Genetic Algorithm (GA) or Evolutionary Programming (EP) and other intelligent algorithms were combined to optimize the parameters of controller, the control performance was greatly improved. As the innovation of specific applications, the stability and universality of these methods need to be further studied and verified.

Moreno-Valenzuela J. and others studied the application of robust control [ $\underline{4}, \underline{23}, \underline{24}]$. The main idea was to ensure that the robot system be stable and maintain a certain performance indice within a certain range of uncertainties. Robust control is a kind of fixed control and is easier to implement. The design of the general robust control system is based on the worst situation, so the system generally does not work in the optimal state.

Topalov A.V. and others studied the application of adaptive control methods to deal with the nonlinear and time-varying problem of industrial robot[25, 26]. The method continuously monitors the characteristics and environment information of the controlled object during operation, automatically changes the parameters and structure of the controller, and adjusts the control function automatically, so as to ensure the system achieves ideal control quality.

Variable structure sliding mode control is a method with high attention in recent years. Ajwad S. and A. Jasim, I. F studied the variable structure sliding mode control in the application of industrial 
robots[24, 27], aiming at the nonlinearity, time-varying parameters and external disturbances. The accurate mathematical model is not a necessity. It drives the state run in a sliding surface to achieve accurate trajectory tracking control of the system.

The iterative learning control theory was proposed and applied to the trajectory tracking of industrial robot by Aromoto et al. [28]. This method imitates human learning process without relying too much on the dynamic model of the robot. It constructs the learning rule according to the previous or current output of the robot object, so that the robot could track the trajectory accurately in a limited time. On this basis, the methods of iterative learning and mathematical models, neural network [29], adaptive control [ $\underline{30}]$, decoupling and dynamic compensation $[\underline{31}, \underline{32}]$ were also studied.

The trajectory tracking control of industrial robots has been widely studied by scholars with various theories and methods. However, the real-time control has not been well solved [33], especially in the case where higher response speed, tracking precision are explicitly specified.

\section{research direction and development trend}

As indicated in section4, due to the complex characteristics of industrial robot and the diversity of application requirements, motion control based on parameter identification, trajectory planning and trajectory tracking of industrial robots is still the basis and key problem for the application of industrial robot[3] . In a sense, the performances of motion control determine the design level and application scope of the robot. The researchers all over the world have been carrying out researches on this issue for decades. Moreover, with the expansion of the range of application and the improvement of control requirements, the study of this problem will continue in the next few decades.

In parameter identification, the traditional methods of detection and identification are deficient in the design, applicability and accuracy. It could be the main research direction to realize fast and reliable online identification by combining optimization and intelligent algorithm.

In trajectory planning, besides to consider the working environment, dynamic characteristics of robot system and the constraints of robot workspace, to achieve the shortest path, minimum energy consumption and smaller jerk should be the main research direction.

In the trajectory tracking control, aiming at the characteristics of nonlinearity, strong coupling, time-varying, uncertainty, the research direction could be applying advanced intelligent control algorithm, neural network, adaptive sliding mode, and iterative learning to realize stable and reliable trajectory that meet the requirements of speed and precision indices.

\section{Conclusion}

Robot is one of the most important technologies in twenty-first Century. Motion control is still the key problem in the research domain. Strengthening the research of basic theory and practice of robot motion control is helpful to improve the performance of industrial robot and expand its application range.

In the study of robot motion control, the parameters identification, trajectory planning and trajectory tracking control are three traditional problems. In this paper, the recent research methods and progress are introduced, and the future direction and development trend of these problems are analyzed. 


\section{Acknowledgment}

This work is supported under the projects of Chinese National Sciences Foundation (No. 61463003 , 51409047), the Chinese Scholarship Council(No.201508360120) and project from Jiangxi Province (JXNE2017-01).

\section{References}

[1] Yuqing, H., Z. Yiwen, and H. Jianda, The new trend of robot technology development. Robot industry, 2015. 2015(5): p. 74-80.

[2] Huixin, S., Report on Industrial Robots Market with Number. Automation Panorama, 2016. 2016(9): p. 28-29.

[3] Min, T. and W. Shuo, Progress in robotics research. Acta Automatica Sinica, 2013. 30(7): p. 963-972.

[4] Jin, J.F. and N. Gans, Parameter identification for industrial robots with a fast and robust trajectory design approach. Robotics and Computer-Integrated Manufacturing, 2015. 31: p. 21-29.

[5] Wilson, J., M. Charest, and R. Dubay, Non-linear model predictive control schemes with application on a 2 link vertical robot manipulator. Robotics and Computer-Integrated Manufacturing, 2016. 41: p. 23-30.

[6] Ajwad, S.A., et al., A systematic review of current and emergent manipulator control approaches. Frontiers of Mechanical Engineering, 2015. 10(2): p. 198-210.

[7] Nandhakumar, S., et al., Position control of industrial robotic manipulator using variable structure control system with single term Haar wavelet series method. Journal of Vibration and Control, 2015. 21(12): p. 2465-2483.

[8] Wu, J., J.S. Wang, and Z. You, An overview of dynamic parameter identification of robots. Robotics and Computer-Integrated Manufacturing, 2010. 26(5): p. 414-419.

[9] Hph, A., inverse dynamic model identification of 2-axes PAM robot arm using neural MIMO NARX model. INtelligent Mechatronics, 2009: p. 1282-1287.

[10]Bingul Z, O. and K. O, dynamic identification of staubli RX-60 robot using PSO and LS methods. Expert Systems with Applications, 2010. 8(4): p. 4136-4149.

[11]Koker, R., A genetic algorithm approach to a neural-network-based inverse kinematics solution of robotic manipulators based on error minimization. Information Sciences, 2013. 222: p. 528-543.

[12]Yan, D.P., Y.Z. Lu, and D. Levy, Parameter Identification of Robot Manipulators: A Heuristic Particle Swarm Search Approach. Plos One, 2015. 10(6).

[13]Tondu, B. and S.A. Bazaz, The three-cubic method: An optimal online robot joint trajectory generator under velocity, acceleration, and wandering constraints. International Journal of Robotics Research, 1999. 18(9): p. 893-901.

[14]Constantinescu, D. and E.A. Croft, Smooth and time-optimal trajectory planning for industrial manipulators along specified path. Journal of Robotic Systems, 2000. 17(5): p. 233-249.

[15]Cho, B.H., B.S. Choi, and J.M. Lee, Time-optimal trajectory planning for a robot system under torque and impulse constraints. International Journal of Control Automation and Systems, 2006. 4(1): p. 10-16. 
[16] Field, G. and Y. Stepanenko. Iterative dynamic programming:anapproachtominimumenergy trajectory planning for roboticmanipulators. in 13th IEEE International Conference on Robotics and Automation. 1996. Min-neapolis,Minn, USA, .

[17]Zha, X.F., Optimal pose trajectory planning for robotmanipulators. Mechanism and MachineTheory, 2002. vol.37(no.10): p. 1063-1086.

[18]Valero, F., V. Mata, and A. Besa, Trajectory planning in workspaces with obstacles taking into account the dynamic robot behaviour Mechanism and MachineTheory, 2006. vol.41(no.5): p. $525-536$.

[19]Benzaoui, M., et al., Trajectory tracking with obstacle avoidance of redundant manipulator based on fuzzy inference systems. Neurocomputing, 2016. 196: p. 23-30.

[20]Corke, P., robotics, vision and control. 2011: Springe.

[21]Y, F. and W. Q., Tracking control of robot manipulators via output feedback linearization. Frontiers of Mechanical Engineering in China, 2006. 1(3).

[22]A, A.S. and Iqbal U, Hardware realization and control of multi-degree of freedom articulated robotic arm. In: Emerging Trends and Applications in Information Communication Technologies, in Communications in Computer and Information Science(CCIS). 2015, Springer: Berlin.

[23] Moreno-Valenzuela, J. and V. Santibanez, Robust Saturated PI Joint Velocity Control for Robot Manipulators. Asian Journal of Control, 2013. 15(1): p. 64-79.

[24]Ajwad, S.A., et al., Disturbance-Observer-Based Robust Control of a Serial-link Robotic Manipulator Using SMC and PBC Techniques. Studies in Informatics and Control, 2015. 24(4): p. 401-408.

[25]Islam, S., et al., Adaptive Control for Robot Manipulators using Multiple Parameter Models. International Journal of Control Automation and Systems, 2016. 14(5): p. 1365-1375.

[26] Topalov, A.V., O. Kaynak, and G. Aydin, Neuro-adaptive sliding-mode tracking control of robot manipulators. International Journal of Adaptive Control and Signal Processing, 2007. 21(8-9): p. 674-691.

[27] Jasim, I.F., P.W. Flapper, and H. Voos, Adaptive sliding mode fuzzy control for unknown robots with arbitrarily-switched constraints. Mechatronics, 2015. 30: p. 174-186.

[28]Zhenhua, Q., Research on trajectory tracking control of industrial robot. 2012, Zhejiang University of Technology.

[29]Ping, J., L. Ziyu, and C. Yangquan, The application of iterative learning neural network control in teaching-learning of robot. Control theory and Application, 2004. 21(3): p. 447-452.

[30]Hang, G., Adaptive iterative learning identification and control for a class of time-varying systems. 2007, Nanjing University of Science and Technology.

[31]Ernesto, H. and J.O. Pedro, Iterative Learning Control with Desired Gravity Compensation under Saturation for a Robotic Machining Manipulator. Mathematical Problems in Engineering, 2015.

[32]Freeman, C.T. and T.V. Dinh, Experimentally verified point-to-point iterative learning control for highly coupled systems. International Journal of Adaptive Control and Signal Processing, 2015. 29(3): p. 302-324.

[33]Xiaohong, H. and L. Cuiming, An iterative learning method for trajectory tracking control of robot. Sciences, Technology and Engineering, 2008. 8(7): p. 1813-1819. 